\title{
ENCEPHALITIS
}

\section{IMMUNOMODULATORY THERAPY FOR RASMUSSEN SYNDROME}

Investigators from the National Epilepsy Center, Shizuoka, Gifu University, Tokyo Women's Medical University, National Center of Neurology and Psychiatry, and Okayama University School of Medicine, Japan, examined seizure, cognitive, and motor outcomes in 49 patients referred with Rasmussen encephalitis (RS) and recently had received initial immunomodulatory therapy: regular IV immunoglobulin (IVIg) at a dose of $100 \mathrm{mg} / \mathrm{kg} / \mathrm{day}$, methylprednisolone steroid pulse therapy at a dose of $30 \mathrm{mg} / \mathrm{kg} / \mathrm{day}$ (children) for 3 days, and tacrolimus $0.1 \mathrm{mg} / \mathrm{kg} /$ day. Mean age at onset was $8.7+/-10.5$ years. Seizure-free rate was $71 \%$ after functional hemispherectomy $(\mathrm{FH})$, seizure response rate was $81 \%$ for regular steroid pulse therapy, $42 \%$ for tacrolimus therapy, and $23 \%$ for regular IVIg therapy. IQ higher than 80 was attained by $50 \%$ of patients treated with steroid pulse therapy, in $43 \%$ following IVIg therapy, in $29 \%$ after tacrolimus therapy, and $0 \%$ in patients treated surgically by FH. In patients without MRI lesions compared to those with advanced lesions, percent of patients with IQ $>80$ were $100 \%$ cf. $37 \%$. Motor paresis was improved only in immunomodulatory treated patients. Motor function was aggravated in $100 \%$ patients treated by FH, $62 \%$ by regular IVIg, and $10 \%$ by regular steroid pulse therapy. (Takahashi Y, Yamazaki E, Mine J, et al. Immunomodulatory therapy versus surgery for Rasmussen syndrome in early childhood. Brain Dev 2013 Sep;35(8):778-85). (Response: Dr Y Takahashi, National Epilepsy Center, Shizuoka, Japan. E-mail: takahashi-ped@umin.ac.jp).

COMMENT. The authors conclude that early initiation of regular steroid pulse therapy is the recommended first-line immunomodulatory therapy in patients with suspect RS. Regular pulse therapy yields superior seizure outcome and better cognitive outcome in early stage RS, before the appearance of MRI lesions. Functional hemispherectomy may be indicated in patients with neurological deficits equivalent to those that would result from surgery, mainly in patients with disease involving the nondominant hemisphere.

Diagnostic criteria for Rasmussen encephalitis. Investigators at Boston Children's Hospital studied the sensitivity, specificity, and positive and negative predictive values of the Bien diagnostic criteria for RE, using pathology findings for diagnosis (Olson HE, et al. Epilepsia 2013 Aug 23 [Epub ahead of print]). In 82 patients evaluated for RE, the Bien criteria had a sensitivity of $81 \%$, with 4 false negatives and 5 false positives.

The Bien diagnostic criteria are met if either all 3 criteria of Part A or two of 3 criteria of Part B are present. Part A criteria are clinical (focal seizures, with or without epilepsia partialis continua), EEG (unihemispheric slowing with or without epileptiform activity), and MRI (unihemispheric focal cortical atrophy). Part B criteria are clinical (epilepsia partialis continua), MRI (progressive unihemispheric focal cortical atrophy) and histopathology ( $\mathrm{T}$ cell dominated encephalitis and reactive astrogliosis). (Bien $\mathrm{CG}$, et al. Pathogenesis, diagnosis and treatment of Rasmussen encephalitis: a European consensus statement. Brain 2005 Mar; 128(Pt 3):454-71). 